\title{
Cerebellar transcranial direct current stimulation improves adaptive postural control
}

Peter Poortvliet ${ }^{1,2}$, Billie Hsieh ${ }^{4}$, Andrew Cresswell ${ }^{1}$, Jacky Au ${ }^{3}$, Marcus Meinzer ${ }^{4}$

${ }^{1}$ The University of Queensland, Centre for Sensorimotor Performance, School of Human Movement and Nutrition Sciences, Brisbane 4072, Australia

2 The University of Queensland, Asia-Pacific Centre for Neuromodulation, Queensland Brain Institute, Brisbane, 4072, Australia

3 The University of California Irvine, Working Memory and Plasticity Lab, Department of Education, Irvine, 92697, United States

${ }^{4}$ The University of Queensland, Centre for Clinical Research, Herston, 4029, Australia

\section{Correspondence:}

Dr. Peter Poortvliet

The University of Queensland

Centre for Sensorimotor Performance

School of Human Movement and Nutrition Sciences

Brisbane, QLD 4072, Australia

Tel: +61733655240

Fax: +61 733656877

e-mail: peterpoortvliet@yahoo.com

Keywords: Standing Postural Balance; Center of Pressure; Cerebellum; Transcranial Direct Current Stimulation; Achilles Tendon Vibration. 


\section{Abstract}

\section{Objective}

Rehabilitation interventions contribute to recovery of impaired postural control, but it remains a priority to optimize their effectiveness. A promising strategy may involve transcranial direct current stimulation (tDCS) of brain areas involved in fine-tuning of motor adaptation. This study explored the effects of cerebellar tDCS (ctDCS) on postural recovery from disturbance by Achilles tendon vibration.

\section{Methods}

Twenty-eight healthy volunteers participated in this sham-ctDCS controlled study. Standing blindfolded on a force platform, four trials were completed: 60 sec quiet standing followed by $20 \mathrm{~min}$ active (anodal-tDCS, $1 \mathrm{~mA}, 20 \mathrm{~min}, \mathrm{~N}=14$ ) or shamctDCS (40 sec, N=14) tDCS; three quiet standing trials with $15 \mathrm{sec}$ of Achilles tendon vibration and $25 \mathrm{sec}$ of postural recovery. Postural steadiness was quantified as displacement, standard deviation and path derived from the center of pressure (COP).

\section{Results}

Baseline demographics and quiet standing postural steadiness, and backwards displacement during vibration were comparable between groups. However, activetDCS significantly improved postural steadiness during vibration and reduced forward displacement and variability in COP derivatives during recovery.

\section{Conclusions}

We demonstrate that ctDCS results in short-term improvement of postural adaptation in healthy individuals.

\section{Significance}

Future studies need to investigate if multisession ctDCS combined with training or rehabilitation interventions can induce prolonged improvement of postural balance. 


\section{Highlights}

- Rehabilitation interventions for recovery of postural control are time consuming and costly.

- Offline cerebellar tDCS can improve postural steadiness in a healthy population.

- These findings may have implications for optimizing rehabilitation interventions in clinical settings. 


\section{Introduction}

Adaptive postural control is essential for almost all aspects of every day life. Impaired postural control results in substantial functional limitations in advanced age (Maki and McIlroy, 1996) and in pathological ageing conditions like stroke (Beyaert et al., 2015), Parkinson's disease (Schoneburg et al., 2013) or multiple sclerosis (Huisinga et al., 2012). Although rehabilitation and conditioning programs have shown promising results in recovery of postural control, those interventions are typically time and cost intensive and may only yield moderate effects (HowardWilsher et al., 2016, Smania et al., 2011, Yitayeh and Teshome, 2016). Optimizing the effectiveness of such interventions by reducing the time and effort required to achieve beneficial outcomes is therefore of utmost importance for aging societies worldwide.

Postural stability and balance are supported by the dynamic interplay of spinal, supra-spinal, subcortical and cortical neural mechanisms (Jacobs and Horak, 2007). Among those, the cerebellum contributes to adaptive postural control by finetuning motor programs (Ioffe et al., 2007). This is particularly important during task conditions that require trial-and-error adaptation of motor behaviors (Diedrichsen et al., 2005, Ito, 2002) or when the postural system is challenged by altered sensory inputs (Winter, 1995) or neurological injury (Grimaldi et al., 2016, Horak and Diener, 1994). Consequently, substantial interest has recently emerged to investigate whether cerebellar function can be enhanced by means of transcranial direct current stimulation (Ferrucci et al., 2015, Grimaldi et al., 2014).

tDCS involves delivering a weak electrical current to the brain via scalp attached electrodes. The current modulates neural excitability at the stimulation site (Stagg and Nitsche, 2011), but also affects functionally connected distant brain regions (Meinzer et al., 2013, Polania et al., 2011). tDCS has a beneficial safety profile (Fregni et al., 2015) and has been shown to allow promotion of long-lasting enhancements of learning and neuroplasticity in health and disease (Cohen Kadosh et al., 2010, Meinzer et al., 2016, Meinzer et al., 2014, Reis et al., 2009). Moreover, a number of previous studies have demonstrated that tDCS administered to cortical sites, like the primary motor or prefrontal cortex, can enhance postural control in healthy individuals (Dutta et al., 2014, Zhou et al., 2014) and in patients with cerebral palsy, Parkinson's disease and stroke (Fregni et al., 2006, Grecco et al., 2017, Kaski et al., 2014, Saeys et al., 2015) However, while previous studies have demonstrated 
that cerebellar tDCS (ctDCS) can improve motor learning in healthy individuals (e.g., Block and Celnik, 2013, Galea et al., 2009, Hardwick and Celnik, 2014), potential beneficial effects on postural control have not yet been investigated. Such an approach would be of particular interest as an adjunct treatment approach in patients with cerebral lesions where clinicians are interested to facilitate activity in spared cortical regions that rely on cerebellar inputs (Grimaldi et al., 2014). Furthermore, ctDCS could significantly improve the responsiveness to balance training and reduce the duration of standard rehabilitation programs (Yitayeh and Teshome, 2016), which may in turn improve adherence and therapeutic outcomes.

The purpose of the present study was to investigate for the first time whether ctDCS can improve postural recovery in response to repeated postural perturbations in a healthy population. Specifically, we manipulated proprioceptive input during upright standing using bilateral Achilles tendon vibration, which is known to result in an inappropriate, gradual 'corrective' backward body movement and a more abrupt recovery of balance after cessation of the vibration (Ceyte et al., 2007, Lackner and Levine, 1979, Winter, 1995). Both postural reactions are mediated by means of sensory feedback mechanisms and anticipatory postural adjustments (Ioffe et al., 2007, Yanagihara, 2014). The primary hypothesis of the present study was that postural adaptation during vibration and after Achilles tendon vibration would improve over subsequent trials, but that this improvement would be greater in the active (i.e., anodal) ctDCS group compared to the placebo (sham) group. We also hypothesized to observe more pronounced effects of ctDCS during the more challenging recovery phase.

\section{Methods}

\subsection{Study overview}

This study assessed the impact of bilateral active ctDCS on standing balance in a randomized, sham controlled, double-blind, between subjects design in healthy young individuals. Across three consecutive experimental trials, participants received bilateral Achilles tendon vibration while being blindfolded and standing on a force platform (Figure 1A). Active or sham ctDCS was administered for $20 \mathrm{~min}$ in a seated position prior to the start of the experimental phase. Potential beneficial offline effects of anodal ctDCS on postural balance during and following the vibration phase were 
explored by comparison of postural performance of the active and sham groups. All procedures were in accordance with the Helsinki Declaration and had been approved by the Research Ethics Committee of the University of Queensland.

\section{- insert Figure 1 here -}

\subsection{Participants}

Twenty-eight healthy adults volunteered to participate in this study and were randomly assigned to the two stimulation conditions (14 per group based on 28 predefined codes that triggered either active or sham cerebellar tDCS). This resulted in two participant groups that were comparable for baseline demographic variables and balance and activity measures (see results section for details). Participants provided signed informed consent prior to study inclusion and were compensated with AU\$50 upon completion of the study.

\subsection{Experimental set-up and procedures}

Participants attended a single experimental session during which they were asked to stand barefoot on a force platform (AMTI, force-sensing treadmill, Watertown, MA) in a natural position with the feet hip-width apart (Figure 1A). Foot positions on the force-platform were marked using tape for appropriate repositioning across trials. Participants were instructed to stand relaxed with their head upright, their gaze straight ahead and their arms at their sides.

All sessions started with $60 \mathrm{~s}$ of blindfolded quiet standing without any vibration in order to assess baseline postural steadiness. Subsequently, $20 \mathrm{~min}$ of active or sham cerebellar tDCS were administered (for details see below), after which participants were repositioned on the force platform for the experimental trials. Bilateral Achilles tendon vibration was administered during the three experimental trials using two custom built mechanical vibrating devices, consisting of small DC motors with eccentric masses contained within a cylindrical PVC housing. The devices were attached to the skin with double sided tape and secured in place using foam cuffs surrounded by Velcro straps. For consistency, the devices were attached to the ankles at baseline and during the three vibration trials, but were only activated during the latter producing a vibration frequency of $75 \mathrm{~Hz}$ with an amplitude of 1.5 $\mathrm{mm}$. 
The pre-vibration phase was kept variable (8-15 s) to ensure that the onset of the vibration was unpredictable and to prevent for anticipatory postural strategies. The following vibration (VIB) phase lasted for $15 \mathrm{~s}$, which was followed by $25 \mathrm{~s}$ of recovery of quiet stance (REC). Participants were blindfolded for the duration of the experimental trials (i.e., pre, during and after vibration). However, the blindfold was temporarily removed during short breaks (1-2 min) between each vibration session, during which the participants remained on the force platform.

Postural control during standing is mostly referred to as the ability to minimize postural sway during quiet standing as well as to maintain balance during posturally challenging conditions (Winter, 1995). Although independent from the center of mass, the center of pressure $(\mathrm{COP})$ can be considered a surrogate for the trajectory of the center of mass during quiet stance (Winter et al., 1990). Postural balance was therefore quantified as the $\mathrm{COP}$ as derived from the $3 \mathrm{D}$ ground reaction forces and moments from the force platform. Data were sampled at $2000 \mathrm{~Hz}$ using a 14-bit analogue-to-digital converter with Qualisys Track manager data acquisition software (Qualisys AB, Gothenburg Sweden).

\subsection{Transcranial direct current stimulation}

A constant direct current was administered using a one-channel direct current stimulator (NeuroConn DC-Stimulator Plus ${ }^{\mathrm{TM}}$ ) with study mode enabled for double blinding. Following the baseline trial of quiet standing, participants of both groups were seated in a chair for 20 minutes to receive either active or sham cerebellar tDCS prior to the subsequent vibration and recovery phases. Offline ctDCS protocols like the one used in our study have previously yielded positive results in healthy individuals and patient populations (for review see van Dun et al. (2017)). More importantly, the equipment could be removed from the head of the participants prior to the experimental trials to prevent possible equipment damage in case of falls.

In both conditions, the current was ramped up to $1 \mathrm{~mA}$ over $10 \mathrm{~s}$, which induces an initial tingling sensation on the scalp to assure blinding of the participants (Gandiga et al., 2006). While the majority of ctDCS studies have used higher stimulation intensities, significant neurophysiological and behavioral effects have also been demonstrated with $1 \mathrm{~mA}$ (van Dun et al., 2016). The stimulation was ramped down over $10 \mathrm{~s}$ in both conditions (after $20 \mathrm{~min}$ in the active ctDCS group and after $40 \mathrm{~s}$ in the sham ctDCS group). The stimulation was administered using two 
conductive rubber electrodes that were inserted in saline solution soaked sponge pockets (anode: $5 \times 7 \mathrm{~cm}^{2}$, cathode: $10 \times 10 \mathrm{~cm}^{2}$ ). The large size of the reference electrode renders it functionally inert without compromising the effects under the anode (Stagg and Nitsche, 2011). The anode was positioned over the cerebellum with the center of the electrode located approximately $1.5 \mathrm{~cm}$ below the inion (Ferrucci et al., 2015). The reference electrode was positioned centrally on the forehead to avoid lateralized current flow. Please note, this project did not have access to a multichannel stimulator which would have allowed for using two anodes and cathodes over the left and right cerebellum and supraorbital cortex. To finalize the experiment, participants were asked to guess whether they had received active or sham cerebellar tDCS.

In order to provide computational support for the effectiveness of cerebellar stimulation using the electrode montage in this study, we modelled electric field intensity (EFI) using the COMETS2 Toolbox. This toolbox allows to generate hexahedral electrode pads which simulate sponge-type electrodes of different sizes and angles and estimate EFI based on a realistic human head model composed of scalp, skull, cerebrospinal fluid, and brain (Lee et al., 2017).

\subsection{Questionnaires and rating scales}

Each experimental session started with completion of a tDCS Safety Screening Form and a set of rating scales assessing physical activity levels (Rapid Assessment of Physical Activity, RAPA (Topolski et al., 2006)), current fatigue levels (11-point visual analogue scale, VAS, $0=$ no-fatigue to $10=$ severely fatigued) and current mood status (mean positive and negative mood ratings; Visual Analogue Mood Scale, VAMS, Folstein and Luria (1973)). Following the last trial, participants were asked to complete the fatigue and mood scales again as well as an adverse effects questionnaire (Brunoni et al., 2011). The latter assessed ten common adverse effects reported in tDCS studies (i.e., headache, tingling, itching, head and scalp pain, burning, sleepiness, problems concentrating and mood changes) on a scale from 1-4 ( 1 =absent, $2=$ mild, $3=$ moderate, $4=$ severe $)$.

\subsection{Data analysis}

Bilateral Achilles tendon vibration predominantly results in a pronounced backward movement, followed by a rapid forward recovery movement upon 
termination of vibration (Lackner and Levine, 1979, Winter, 1995). Therefore, our analyses focused on movements in the sagittal plane. All experimental data were processed and analyzed offline using MATLAB. Data were first low pass filtered using a $4^{\text {th }}$ order Butterworth filter with a cut-off frequency of $20 \mathrm{~Hz}$ and then down sampled to $200 \mathrm{~Hz}$. All data were normalized to the average COP during the last five seconds of quiet standing prior to the start of the Achilles tendon vibration trial (i.e., mean signal ${ }_{\text {during vibration }}-$ mean signal $\left.l_{\text {pre-vibration }}\right)$. Data analysis focused on the baseline, vibration and recovery sections of all trials (Figure 1B). We used three of the most commonly used COP parameters to investigate postural control that also have good test-retest reliability (Panzer et al., 2011, Ruhe et al., 2011, Takacs et al., 2014). COP displacement $\left(\mathrm{COP}_{\mathrm{DISP}}\right)$ reflects body excursion/inclination; i.e., the magnitude of deviation of the center of pressure (forward or backward). Less deviation indicates better postural balance (Panzer et al., 2011). The other two, standard deviation $\left(\mathrm{COP}_{\mathrm{SD}}\right)$ and total path length $\left(\mathrm{COP}_{\mathrm{PATH}}\right)$ provide information about the variability during the excursion or sway, or the steadiness of the COP signal (Takacs et al., 2014). Smaller values for all of these parameters are proposed to indicate greater postural stability (Palmieri et al., 2002).

\subsection{Statistical analysis}

Demographic characteristics of the participants, baseline quiet standing data of and degree of adverse effects were compared in the two stimulation groups using twosample t-tests or $\mathrm{Chi}^{2}$-tests as appropriate. Repeated measures ANOVAs compared data acquired during the vibration and recovery phases in both stimulation conditions with the between subjects factor, STIMULATION and the within factor TIME (i.e., three consecutive experimental trials). To assess potential changes in mood, means of positive (VAMS $\mathrm{POS}_{\mathrm{P}}$ : energetic, happy) and negative $\left(\mathrm{VAMS}_{\mathrm{NEG}}\right.$ : afraid, confused, sad, tired, angry, tense) scales and fatigue levels were compared within (paired t-tests) and between groups (two-sample t-test on differences between pre-post assessments). Statistical significance level was set at $\mathrm{P}<0.05$. Data are expressed as mean $\pm \mathrm{SD}$ in text and figures.

\section{Results}

\subsection{Demographics and questionnaires}


The two stimulation groups were matched for gender (sham- vs. activecerebellar tDCS: 7 vs. 9 females, 7 vs. 5 males, p=0.46), age (25.14 \pm 4.44 vs. $25.64 \pm$ 3.82 years, $\mathrm{P}=0.75)$, and body mass index $\left(23.87 \pm 4.03\right.$ vs. $23.31 \pm 5.84 \mathrm{~kg} / \mathrm{m}^{2}$, $\mathrm{P}=0.77)$. In addition, baseline physical activity measures (11.50 \pm 5.93 vs. $11.85 \pm$ 4.47, $\mathrm{P}=0.85$ ) did not differ between the two groups. All participants tolerated the stimulation well and only mild adverse effects were observed. No significant differences were found between the stimulation groups, except for mild tingling, which was rated as more pronounced in the active cerebellar tDCS group (see Table 1 for details).

\section{- insert Table 1 here -}

The type of stimulation was guessed correctly at the end of the session by $6 / 14$ participants in the sham group and by 6/14 participants in the active group. Thus, despite more pronounced mild tingling in the active group, the proportion of correct guesses was identical and below chance level in both groups. Fatigue levels were not different before and following conclusion of the experimental sessions in the two groups, as were changes over time (pre/post sham vs. active: $3.35 \pm 2.82 / 3.07 \pm 2.43$ vs. $2.89 \pm 1.55 / 2.39 \pm 1.71$, all $\mathrm{P}=0.26-0.67)$.

No changes in positive and negative mood ratings were observed in the two stimulation groups (pre/post sham vs. active cerebellar tDCS VAMS $24.78 / 41.14 \pm 25.67$ vs. $47.57 \pm 28.49 / 43.85 \pm 29.62$; VAMS $_{\mathrm{NEG}}: 15.55 \pm 12.57 / 10.28$ \pm 8.58 vs. $10.29 \pm 8.06 / 8.28 \pm 9.75$, all $\mathrm{P}=0.06-0.75)$. The change from pre-to post mood ratings was also comparable between stimulation conditions for both positive and negative mood ratings $(\mathrm{P}=0.27-0.79)$.

\subsection{Postural steadiness}

\subsubsection{Baseline trial}

During the initial $60 \mathrm{~s}$ of quiet standing without ankle vibration, both groups showed similar postural steadiness over time (Figure 2, first column, $\mathrm{P}=0.49,0.38$ and 0.58 respectively).

\section{- insert Figure 2 here -}




\subsubsection{Vibration phase}

Both groups showed an immediate disturbance of quiet standing at the onset of vibration, quantified by a gradual posterior shift in $\mathrm{COP}_{\mathrm{DISP}}$, which persisted during the entire $15 \mathrm{~s}$ of vibration (Figure 1B illustrates a typical trial) that was similar between groups $(\mathrm{F}(1.26)=2.00, \mathrm{P}=0.16$, Figure $2 \mathrm{~A}$, middle column). Despite the marked disturbance to quiet standing, all participants managed to complete the trials without stepping or falling.

The impact of the disturbance gradually decreased across the three consecutive trials in both groups for all parameters (Figure 2A-C, middle column) as a result of adaptive learning (main effect TIME: $\mathrm{F}(2,52)=7.53 / 15.94 / 12.03$, all $\mathrm{P}<0.01$ for $\mathrm{COP}_{\mathrm{DISP}}, \mathrm{COP}_{\mathrm{SD}}$ and $\mathrm{COP}_{\mathrm{PATH}}$ respectively). Nonetheless, the vibration resulted in a statistically significant postural disturbance across all COP parameters and time points in both stimulation groups (all $\mathrm{t}(13)>7.05$, all $\mathrm{p}<.0001$ ). Although there was no difference in $\mathrm{COP}_{\mathrm{DISP}}$ between the two groups, $\mathrm{COP}_{\mathrm{SD}}$ and $\mathrm{COP}_{\mathrm{PATH}}$ were significantly smaller in the active group across all three trials (main effect STIMULATION: both $\mathrm{F}(1,26)>7.75, \quad \mathrm{P}<0.01)$. TIME and STIMULATION interactions were not significant (all $\mathrm{P}>0.23$ ).

\subsubsection{Recovery phase}

Cessation of Achilles tendon vibration resulted in a rapid and immediate recovery response, quantified by an anterior shift in COP that significantly overshot the COP observed during quiet standing prior to vibration (see Figure 1B for a typical example). This immediate anterior overshoot was followed by an oscillating recovery sway pattern that gradually resided. Although both groups showed similar posterior $\mathrm{COP}_{\mathrm{DISP}}$ during the vibration phase, the immediate anterior $\mathrm{COP}_{\mathrm{DISP}}$ as a result of termination of vibration significantly differed between groups (Figure 2A, right column) and was smaller in the active group (STIMULATION: $F(1,26)=6.77$, $\mathrm{P}<0.05)$. Similarly, $\mathrm{COP}_{\mathrm{SD}}$ and $\mathrm{COP}_{\mathrm{PATH}}$ were significantly lower in the active group $(\mathrm{P}<0.05$ and $\mathrm{P}<0.01$, respectively) reflecting a smaller impact of the disturbance to postural steadiness to the active group (Figure 2B and C, right column). Performance of postural steadiness improved across trials in both groups (TIME: $F(2.52)=$ 12.08/8.39/8.09, all parameters $\mathrm{P}<0.01$, for $\mathrm{COP}_{\mathrm{DISP}}, \mathrm{COP}_{\mathrm{SD}}$ and $\mathrm{COP}_{\mathrm{PATH}}$ respectively). None of the interactions between TIME and STIMULATION were significant (all $\mathrm{P}=0.10-0.58)$. 


\subsection{Current modeling}

Current modeling demonstrated that the montage used in the present study effectively targeted the cerebellum (see Figure 3 for details). The intensity of the induced electric field was maximal underneath and adjacent to the anode affecting primarily ventral and dorsolateral aspects of the cerebellum and the cerebellar vermis, with some spread to occipito-parietal regions. Additional effects were observed at the edges of the cathode, with highest EFI in the ventral prefrontal cortex.

\section{- insert Figure 3 here -}

\section{Discussion}

The present study investigated for the first time the potential beneficial effects of anodal cerebellar tDCS on the control of adaptive postural balance during and immediately after a sensory perturbation in young healthy individuals. We demonstrate that the active cerebellar tDCS group performed significantly better in regaining and maintaining standing postural steadiness compared to the group that had received sham tDCS, both during the vibration phase and also the subsequent recovery phase.

Achilles tendon vibration during quiet standing is an established paradigm to investigate adaptive postural control. Adaptation in response to the sensory perturbation typically results in gradual sensorimotor adaptation and improvement of performance indicated by decreased magnitudes of different COP parameters (Ceyte et al., 2007, Paillard and Noé, 2015). In the present study, both groups showed gradual improvement in standing balance performance during and following vibration across the three consecutive trials, which illustrates this adaptive process. Importantly, displacement in response to Achilles tendon vibration showed the weakest behavioral adaptation across trials in both groups, which suggests that the initial posterior displacement is largely driven by the abrupt and strong sensory disturbance. Contrary, cessation of the vibration stimulus only requires a corrective postural response (i.e., a system inherent process involving the cerebellum). Therefore, it is not surprising that ctDCS had stronger effects during the recovery phase, i.e., when additional sensory input had ceased. Moreover, despite similar gross displacement in the two stimulation 
groups, lower path length $\left(\mathrm{COP}_{\mathrm{PATH}}\right)$ and reduced variability $\left(\mathrm{COP}_{\mathrm{SD}}\right)$ in the active ctDCS group suggest better fine tuning of postural control already during the vibration phase, which was maintained during the recovery phase.

Notably, participants in the sham ctDCS group showed the expected pattern of gradual improvement due to learning over consecutive vibration and recovery trials. On the other hand, participants in the active ctDCS group showed significantly lower and more consistent COP values already during the first trial and this effect was maintained during subsequent trials. Moreover, the sham ctDCS group only reached the performance level of the active ctDCS group during the last of the three trials. This finding is in line with previous studies that demonstrated significantly improved locomotor and visuo-motor adaptation to perceptual disturbances with anodal compared sham ctDCS (Block and Celnik, 2013, Galea et al., 2009, Hardwick and Celnik, 2014, Jayaram et al., 2012). The faster and more efficient postural adaptation induced by active stimulation, warrants exploration of this type of intervention in combination with behavioral training in elderly individuals or patient populations with impaired postural balance (e.g., Parkinson's disease or stroke). It needs to be noted that several recent studies failed to find positive effects of ctDCS on postural control (e.g., Steiner et al. 2016) or reported positive effects of inhibitory cathodal ctDCS (e.g., Inukai et al. 2016). However, the protocol used in the latter study substantially deviated from our study (e.g., regarding stimulation parameters, outcome measures, visual cues) and only assessed ctDCS effects on quiet standing performance (i.e., no disturbance requiring adaptive postural control was investigated). Likewise, while the study by Steiner et al. (2016) involved learning of a complex motor skill, the authors speculated that their healthy young subjects may have performed at "maximum possible learning level", which may have prevented further improvement by ctDCS. Nonetheless, these studies highlight that multiple factors that are currently not well understood mediate ctDCS response (van Dun et al., 2016).

An important aspect in both experimental and clinical tDCS studies is the timing of the stimulation with regard to the intervention. For example, beneficial effects of motor cortex tDCS have been demonstrated when the stimulation was administered either prior to (Cabral et al., 2015) or during task performance (Nitsche et al., 2003, Stagg et al., 2011). Similarly, cerebellar tDCS has been shown to impact motor behavior or cognition when administered prior to or during task performance 
(for review (van Dun et al., 2016)). With regard to other types of motor adaptation, the majority of studies reporting positive behavioral stimulation effects administered ctDCS during task performance, thereby exploring online effects of the stimulation (e.g., Galea et al., 2009, Hardwick and Celnik, 2014, Jayaram et al., 2012). In the present study, we administered tDCS prior to the vibration and recovery phases in a seated position (partly for safety reasons, please see methods section), thereby exploiting the after effects of tDCS. Motor cortex tDCS effects have been demonstrated to outlast the end of the stimulation period by approximately $60-90 \mathrm{~min}$ (Nitsche et al., 2007). However, it is currently unknown whether the after effects of ctDCS follow the same time course as those observed after motor cortex stimulation. Nonetheless, our results suggest that this approach was sufficient to improve postural adaptation, which is also in line with previous studies demonstrating significant modulation of neurophysiological parameters and behavior when using offline protocols (for reviews see van Dun et al. (2016 and 2017)).

Regarding the underlying neural mechanisms in the present study: A relatively large network of brain regions, including sensory-motor and premotor regions, the basal ganglia and thalamus, fronto-parietal regions and the cerebellum has been implicated in postural control and adaptation to postural disturbance (Lalonde and Strazielle, 2007). Parts of the cerebellum (i.e., anterior and posterior lobe, anterior vermis) and the prefrontal cortex are recruited during task conditions that impose challenges on postural control and require integration of proprioceptive information from the foot and ankle regarding body sway as in the present study (Diedrichsen et al., 2005, Goble et al., 2011, Ito, 2002, Ouchi et al., 1999, Winter, 1995). Here, the cerebellum is thought to mediate compensatory postural reactions by means of sensory feedback loops, but also anticipatory postural adjustments via feedforward mechanisms (Ioffe et al., 2007, Yanagihara, 2014). Importantly, our current modeling results suggest that both the lateral cerebellum and the vermis were directly affected by the current montage.

However, it is important to note that conventional tDCS set-ups that use relatively large rubber pads like the one we used in the present study and, to the best of our knowledge, all previous studies that used ctDCS (for review see van Dun et al. (2017)) do not allow attributing stimulation effects to the target region alone. Indeed, for cortical montages, current spread to neighboring regions or regions in between the active and reference electrode has been demonstrated, as well as neural network 
effects of tDCS in functionally connected regions (Kuo et al., 2013, Meinzer et al., 2012). While ctDCS may result in more focal stimulation of the cerebellum when the return electrode is attached over extra cephalic regions or the cheek (Parazzini et al., 2014, Rampersad et al., 2014), recent functional imaging studies have demonstrated similar widespread modulation of functionally connected brain regions by ctDCS (D'Mello et al., 2017, Turkeltaub et al., 2016). Likewise, the electric field induced by the montage that was used in the present study affected relatively large cerebellar regions, mostly in task-relevant ventrolateral and medial cerebellar regions, which likely resulted in modulation of activity in multiple functionally connected brain regions. Therefore, the exact neural mechanisms underlying our positive behavioral effects need to be determined in the future.

There is also evidence for distinct roles of the cerebellum and the motor cortex in the process of acquisition and retention of adaptive motor learning. For example, Galea et al. (2011) demonstrated that cerebellar tDCS caused faster visuomotor adaptation, resulting in a rapid reduction in reaching movement errors. Stimulation of the primary motor cortex, however, resulted in augmentation of retention of the learnt adaptation. Our results similarly show an immediate improvement in postural steadiness in the cerebellar tDCS group, indicating rapid acquisition of adaptive motor skills. Future studies are thus required to tease apart the contribution of both regions to long-term maintenance of the immediate gains reported in the present study.

\section{Limitations}

Achilles tendon vibration during standing postural balance is an elegant and well accepted paradigm to study postural adaptation in healthy individuals (Lackner and Levine, 1979). However, the fast habituation to repeated exposure to vibration, which is strongest after the first trial and can last for hours or even days (Rogers et al., 1985, Thompson et al., 2007, Wierzbicka et al., 1998), limits the potential to test performance of postural steadiness in within-subject designs. Similar to previous studies that employed different adaptation paradigms (Hardwick and Celnik, 2014, Panouillères et al., 2015), we opted for a between group design and carefully matched participants for baseline demographic and physical activity variables. Moreover, no differences were found with regard to quiet standing COP parameters prior to tDCS and the gradual posterior displacement was comparable between the two groups. 
Thus, while participants in the two stimulation groups were comparable with regard to those parameters, it needs to be acknowledged that we were unable to assess potential baseline differences in vibration susceptibility. However, the initial backward displacement during vibration was virtually identical in the two groups and the sham ctDCS group showed a gradual pattern of adaptation across trials during recovery, which is typical for the Achilles tendon paradigm. Contrary, the active ctDCS group showed maximal adaptation already on the first trial and maintenance of this effect over time. Those factors argue against the possibility that baseline differences in vibration susceptibility explain our results.

While the present study demonstrated that cerebellar tDCS can improve standing postural balance, the results do not provide insights into the underlying mechanisms of these adaptations. This needs to be scrutinized in future studies using additional neurophysiological measures of motor system excitability. In addition, long-term effects of cerebellar tDCS and interactions with different types of motor adaptation or rehabilitation programs need to be investigated.

\section{Conclusions}

In sum, the results of our study suggest that cerebellar tDCS is suited to induce short-term improvement of postural control even in absence of concurrent motor training. This may have implications for future studies aiming to enhance the effectiveness of training and rehabilitation programs in clinical populations with impaired postural control.

\section{Conflicts of interest}

None.

\section{Acknowledgements}

Financial support was provided by the Australian Research Council (MM: ARC FT120100608) and a University of Queensland internal Research Collaboration Seeding Grant. 


\section{References}

Beyaert C, Vasa R, Frykberg GE. Gait post-stroke: Pathophysiology and rehabilitation strategies. Neurophysiol Clin. 2015;45:335-55. doi: 10.1016/j.neucli.2015.09.005.

Block H, Celnik P. Stimulating the cerebellum affects visuomotor adaptation but not intermanual transfer of learning. Cerebellum. 2013;12:781-93. doi: 10.1007/s12311013-0486-7.

Brunoni AR, Amadera J, Berbel B, Volz MS, Rizzerio BG, Fregni F. A systematic review on reporting and assessment of adverse effects associated with transcranial direct current stimulation. Int J Neuropsychopharmacol. 2011;14:1133-45. doi: $10.1017 /$ S 1461145710001690.

Cabral ME, Baltar A, Borba R, Galvao S, Santos L, Fregni F, Monte-Silva K. Transcranial direct current stimulation: before, during, or after motor training? Neuroreport. 2015;26:618-22. doi: 10.1097/WNR.0000000000000397.

Ceyte H, Cian C, Zory R, Barraud PA, Roux A, Guerraz M. Effect of Achilles tendon vibration on postural orientation. Neurosci Lett. 2007;416:71-5. doi: 10.1016/j.neulet.2007.01.044.

Cohen Kadosh R, Soskic S, Iuculano T, Kanai R, Walsh V. Modulating neuronal activity produces specific and long-lasting changes in numerical competence. Curr Biol. 2010;20:2016-20. doi: 10.1016/j.cub.2010.10.007.

D'Mello AM, Turkeltaub PE, Stoodley CJ. Cerebellar tDCS modulates neural circuits during semantic prediction: A combined tDCS-fMRI study. J Neurosci. 2017;37:1604-13. https://doi.org/10.1523/JNEUROSCI.2818-16.2017

Diedrichsen J, Hashambhoy Y, Rane T, Shadmehr R. Neural correlates of reach errors. J Neurosci. 2005;25:9919-31. doi: 10.1523/jneurosci.1874-05.2005.

Dutta A, Chugh S, Banerjee A, Dutta A. Point-of-care-testing of standing posture with Wii balance board and Microsoft Kinect during transcranial direct current stimulation: a feasibility study. NeuroRehabilitation. 2014;34:789-98. doi: 10.3233/NRE-141077.

Ferrucci R, Cortese F, Priori A. Cerebellar tDCS: How to do it. Cerebellum. 2015;14:27-30. doi: 10.1007/s12311-014-0599-7.

Folstein MF, Luria R. Reliability, validity and clinical application of the visual analogue mood scales. Psychol Med. 1973;3:479-86.

Fregni F, Boggio PS, Santos MC, Lima M, Vieira AL, Rigonatti SP, Silva MT, Barbosa ER, Nitsche MA, Pascual-Leone A. Noninvasive cortical stimulation with transcranial direct current stimulation in Parkinson's disease. Mov Disord. 2006;21:1693-702. doi: 10.1002/mds.21012.

Fregni F, Nitsche MA, Loo CK, Brunoni AR, Marangolo P, Leite J, Carvalho S, Bolognini N, Caumo W, Paik NJ, Simis M, Ueda K, Ekhitari H, Luu P, Tucker DM, 
Tyler WJ, Brunelin J, Datta A, Juan CH, Venkatasubramanian G, Boggio PS, Bikson M. Regulatory considerations for the clinical and research use of transcranial direct current stimulation (tDCS): review and recommendations from an expert panel. Clin Res Regul Aff. 2015;32:22-35. doi: 10.3109/10601333.2015.980944.

Galea JM, Jayaram G, Ajagbe L, Celnik P. Modulation of cerebellar excitability by polarity-specific noninvasive direct current stimulation. J Neurosci. 2009;29:9115-22. doi: 10.1523/JNEUROSCI.2184-09.2009.

Galea JM, Vazquez A, Pasricha N, de Xivry JJ, Celnik P. Dissociating the roles of the cerebellum and motor cortex during adaptive learning: the motor cortex retains what the cerebellum learns. Cereb Cortex. 2011;21:1761-70. doi: 10.1093/cercor/bhq246.

Gandiga PC, Hummel FC, Cohen LG. Transcranial DC stimulation (tDCS): a tool for double-blind sham-controlled clinical studies in brain stimulation. Clin Neurophysiol. 2006;117:845-50. doi: 10.1016/j.clinph.2005.12.003.

Goble DJ, Coxon JP, Van Impe A, Geurts M, Doumas M, Wenderoth N, Swinnen SP. Brain activity during ankle proprioceptive stimulation predicts balance performance in young and older adults. J Neurosci. 2011;31:16344-52. doi:

10.1523/JNEUROSCI.4159-11.2011.

Grecco LA, Oliveira CS, Duarte NA, Lima VL, Zanon N, Fregni F. Cerebellar transcranial direct current stimulation in children with ataxic cerebral palsy: A shamcontrolled, crossover, pilot study. Dev Neurorehabil. 2017;20:142-8.

Grimaldi G, Argyropoulos GP, Bastian A, Cortes M, Davis NJ, Edwards DJ, Ferrucci R, Fregni F, Galea JM, Hamada M, Manto M, Miall RC, Morales-Quezada L, Pope PA, Priori A, Rothwell J, Tomlinson SP, Celnik P. Cerebellar Transcranial Direct Current Stimulation (ctDCS): A novel approach to understanding cerebellar function in health and disease. Neuroscientist. 2016;22:83-97. doi: $10.1177 / 1073858414559409$.

Grimaldi G, Argyropoulos GP, Boehringer A, Celnik P, Edwards MJ, Ferrucci R, Galea JM, Groiss SJ, Hiraoka K, Kassavetis P, Lesage E, Manto M, Miall RC, Priori A, Sadnicka A, Ugawa Y, Ziemann U. Non-invasive Cerebellar Stimulation - a Consensus Paper. Cerebellum. 2014;13:121-38. doi: 10.1007/s12311-013-0514-7.

Hardwick RM, Celnik PA. Cerebellar direct current stimulation enhances motor learning in older adults. Neurobiol Aging. 2014;35:2217-21. doi:

10.1016/j.neurobiolaging.2014.03.030.

Horak FB, Diener HC. Cerebellar control of postural scaling and cental set in stance. J Neurophysiol. 1994;72:479-93.

Howard-Wilsher S, Irvine L, Fan H, Shakespeare T, Suhrcke M, Horton S, Poland F, Hooper L, Song F. Systematic overview of economic evaluations of health-related rehabilitation. Disabil Health J 2016;9:11-25. doi: 10.1016/j.dhjo.2015.08.009.

Huisinga JM, Yentes JM, Filipi ML, Stergiou N. Postural control strategy during standing is altered in patients with multiple sclerosis. Neurosci Lett. 2012;524:124-8. doi: 10.1016/j.neulet.2012.07.020. 
Inukai Y, Saito K, Sasak iR, Kotan S, Nakagawa M, Onishi H. Influence of transcranial direct current stimulation to the cerebellum on standing posture control. Front Hum Neurosci. 2016;10:325. doi: 10.3389/fnhum.2016.00325.

Ioffe ME, Chernikova LA, Ustinova KI. Role of cerebellum in learning postural tasks. Cerebellum. 2007;6:87-94. doi: 10.1080/14734220701216440.

Ito M. Historical review of the significance of the cerebellum and the role of Purkinje cells in motor learning. Ann N Y Acad Sci. 2002;978:273-88. doi: 10.1111/j.17496632.2002.tb07574.x.

Jacobs JV, Horak FB. Cortical control of postural responses. J Neural Transm. 2007;114:1339-48. doi: 10.1007/s00702-007-0657-0.

Jayaram G, Tang B, Pallegadda R, Vasudevan EV, Celnik P, Bastian A. Modulating locomotor adaptation with cerebellar stimulation. J Neurophysiol. 2012;107:2950-7. doi: 10.1152/jn.00645.2011.

Kaski D, Dominguez RO, Allum JH, Islam AF, Bronstein AM. Combining physical training with transcranial direct current stimulation to improve gait in Parkinson's disease: a pilot randomized controlled study. Clin Rehabil. 2014;28:1115-24. doi: $10.1177 / 0269215514534277$.

Kuo H, Bikson M, Datta A, Minhas P, Paulus W, Kuo MF, Nitsche MA. Comparing cortical plasticity induced by conventional and high-definition $4 \times 1$ ring tDCS: a neurophysiological study. Brain Stimul. 2013;6:644-8. doi:

10.1016/j.brs.2012.09.010.

Lackner JR, Levine MS. Changes in apparent body orientation and sensory localization induced by vibration of postural muscles: vibratory myestethetic illusions. Aviat Space Environ Med. 1979;50:346-54.

Lalonde R, Strazielle C. Brain regions and genes affecting postural control. Prog Neurobiol. 2007;81:45-60. doi: 10.1016/j.brainresrev.2011.02.005.

Lee C, Jung YJ, Lee SJ, Im CH. COMETS2: An advanced MATLAB toolbox for the numerical analysis of electric fields generated by transcranial direct current stimulation. J Neurosci Methods. 2017;277:56-62. doi:

10.1016/j.jneumeth.2016.12.008.

Maki BE, McIlroy WE. Postural control in the older adult. Clin Geriatr Med. 1996;12:635-58.

Meinzer M, Antonenko D, Lindenberg R, Hetzer S, Ulm L, Avirame K, Flaisch T, Flöel A. Electrical brain stimulation improves cognitive performance by modulating functional connectivity and task-specific activation. J Neurosci. 2012;32:1859-66. doi: 10.1523/JNEUROSCI.4812-11.2012.

Meinzer M, Darkow R, Lindenberg R, Flöel A. Electrical stimulation of the motor cortex enhances treatment outcome in post-stroke aphasia. Brain. 2016;139:1152-63. doi: 10.1093/brain/aww002. 
Meinzer M, Jahnigen S, Copland DA, Darkow R, Grittner U, Avirame K, Rodriguez AD, Lindenberg R, Floel A. Transcranial direct current stimulation over multiple days improves learning and maintenance of a novel vocabulary. Cortex. 2014;50:137-47. doi: 10.1016/j.cortex.2013.07.013.

Meinzer M, Lindenberg R, Antonenko D, Flaisch T, Flöel A. Anodal transcranial Direct Current Stimulation temporarily reverses age-associated cogntive decline and functional brain activity changes. J Neurosci. 2013;33:12470-8. doi: 10.1523/JNEUROSCI.5743-12.2013.

Nitsche MA, Doemkes S, Karakose T, Antal A, Liebetanz D, Lang N, Tergau F, Paulus W. Shaping the effects of transcranial direct current stimulation of the human motor cortex. J Neurophysiol. 2007;97:3109-17. doi: 10.1152/jn.01312.2006.

Nitsche MA, Schauenburg A, Lang N, Liebetanz D, Exner C, Paulus W, Tergau F. Facilitation of implicit motor learning by weak transcranial direct current stimulation of the primary motor cortex in the human. J Cogn Neurosci. 2003;15:619-26. doi: $10.1162 / 089892903321662994$.

Ouchi Y, Okada H, Yoshikawa E, Nobezawa S, Futatsubashi M. Brain activation during maintenance of standing postures in humans. Brain. 1999;122:329-38.

Paillard T, Noé F. Techniques and methods for testing the postural function in healthy and pathological subjects. BioMed Res Int. 2015;2015:891390. doi:

10.1155/2015/891390.

Palmieri RM, Ingersoll CD, Stone MB, Krause BA. Center-of-pressure parameters used in the assessment of postural control. J Sport Rehabil. 2002;11:51-66. doi: 10.1007/s00586-010-1543-2.

Panouillères MT, Joundi RA, Brittain JS, Jenkinson N. Reversing motor adaptation deficits in the ageing brain using non-invasive stimulation. J Physiol. 2015;593:364555. doi: 10.1113/JP270484.

Panzer VP, Wakefield DB, Hall CB, Wolfson LI. Mobility assessment: Sensitivity and specificity of measurement sets in older adults. Arch Phys Med Rehabil. 2011;92:905-12. doi: 10.1016/j.apmr.2011.01.004.

Parazzini M, Rossi E, Ferrucci R, Liorni I, Priori A, Ravazzani P. Modelling the electric field and the current density generated by cerebellar transcranial DC stimulation in humans. Clin Neurophysiol. 2014;125:577-84. doi:

10.1016/j.clinph.2013.09.039.

Polania R, Nitsche MA, Paulus W. Modulating functional connectivity patterns and topological functional organization of the human brain with transcranial Direct Current Stimulation. Hum Brain Mapp. 2011;32:1236-49. doi: 10.1002/hbm.21104.

Rampersad SM, Janssen AM, Lucka F, Aydin Ü, Lanfer B, Lew S, Wolters CH, Stegeman DF, Oostendorp TF. Simulating transcranial direct current stimulation with a detailed anisotropic human head model. IEEE Trans Neural Syst Rehabil Eng. 2014;22:441-52. doi: 10.1109/TNSRE.2014.2308997. 
Reis J, Schambra HM, Cohen LG, Buch ER, Fritsch B, Zarahn E, Celnik PA, Krakauer JW. Noninvasive cortical stimulation enhances motor skill acquisition over multiple days through an effect on consolidation. Proc Natl Acad Sci U S A. 2009;106:1590-5. doi: 10.1073/pnas.0805413106.

Rogers D, Bendrups A, Lewis M. Disturbed proprioception following a period of muscle vibration in humans. Neurosci Lett. 1985;57:147-52.

Ruhe A, Fejer R, Walker B. Center of pressure excursion as a measure of balance performance in patients with non-specific low back pain compared to healthy controls: a systematic review of the literature. Eur Spine J. 2011;20:358-68. doi: 10.1007/s00586-010-1543-2.

Saeys W, Vereeck L, Lafosse C, Truijen S, Wuyts FL, Van De Heyning P.

Transcranial direct current stimulation in the recovery of postural control after stroke: a pilot study. Disabil Rehabil. 2015;37:1857-63. doi:

10.3109/09638288.2014.982834.

Schoneburg B, Mancini M, Horak F, Nutt JG. Framework for understanding balance dysfunction in Parkinson's disease. Mov Disord. 2013;28:1474-82. doi:

10.1002/mds. 25613.

Smania N, Picelli A, Geroin C, Ianes P, La Marchina E, Zenorini A, Gandolfi M. Balance and gait rehabilitation in patients with Parkinson's disease. In: Rana AQ, editor. Diagnosis and treatment of Parkinson's disease: InTech; 2011, doi $10.5772 / 22561$

Stagg CJ, Jayaram G, Pastor D, Kincses ZT, Matthews PM, Johansen-Berg H. Polarity and timing-dependent effects of transcranial direct current stimulation in explicit motor learning. Neuropsychologia. 2011;49:800-4. doi: 10.1016/j.neuropsychologia.2011.02.009.

Stagg CJ, Nitsche MA. Physiological Basis of Transcranial Direct Current Stimulation. Neuroscientist. 2011;17:37-53. doi: 10.1177/1073858410386614.

Steiner KM, Enders A, Thier W, Batsikadze G, Ludolph N, Ilg W, Timmann D. Cerebellar tDCS does not improve learning in a complex whole body dynamic balance task in young healthy subjects. PLoS One. 2016;11:e0163598.

Takacs J, Carpenter MG, Garland SJ, Hunt MA. Test re-test reliability of centre of pressure measures during standing balance in individuals with knee osteoarthritis. Gait Posture. 2014;40:270-3. doi: 10.1016/j.gaitpost.2014.03.016.

Thompson C, Belanger M, Fung J. Effects of bilateral Achilles tendon vibration on postural orientation and balance during standing. Clin Neurophysiol. 2007;118:245667.

Topolski TD, LoGerfo J, Patrick DL, Williams B, Walwick J, Patrick MB. The rapid assessment of physical activity (RAPA) among older adults. Prev Chronic Dis. 2006;3:A118. 
Turkeltaub PE, Swears MK, D'Mello AM, Stoodley CJ. Cerebellar tDCS as a novel treatment for aphasia? Evidence from behavioral and resting-state functional connectivity data in healthy adults. Restor Neurol Neurosci. 2016;34:491-505. doi: 10.3233/RNN-150633.

van Dun K, Bodranghien FC, Manto M, Mariën P. Targeting the cerebellum by noninvasive neurostimulation: a review Cerebellum. 2017;16:695-741. doi: 10.1007/s12311-016-0840-7.

van Dun K, Bodranghien FC, Marien P, Manto MU. tDCS of the cerebellum: Where do we stand in 2016? Technical issues and critical review of the literature. Front Hum Neurosci. 2016;10:199. doi: 10.3389/fnhum.2016.00199.

Wierzbicka M, Gilhodes J, Roll J. Vibration-induced postural posteffects. J Neurophysiol. 1998;79:143-50.

Winter D. Human balance and posture control during standing and walking. Gait Posture. 1995;3:193-214.

Winter DA, Patla AE, Frank JS. Assessment of balance control in humans. Med Prog Technol. 1990;16:31-51.

Yanagihara D. Role of the cerebellum in postural control. J Phys Fitness Sports Med. 2014;3:169-72. doi: 10.7600/jpfsm.3.169.

Yitayeh A, Teshome A. The effectiveness of physiotherapy treatment on balance dysfunction and postural instability in persons with Parkinson's disease: a systematic review and meta-analysis. BMC Sports Sci Med Rehabil. 2016;8:17-27. 10.1186/s13102-016-0042-0.

Zhou J, Hao Y, Wang Y, Jor'dan A, Pascual-Leone A, Zhang J, Fang J, Manor B. Transcranial direct current stimulation reduces the cost of performing a cognitive task on gait and postural control. Eur J Neurosci. 2014;39:1343-8. 10.1111/ejn.12492. 


\section{Figure legends}

Fig. 1. Overview of the protocol with the experimental trials following the $60 \mathrm{~s}$ of baseline quiet standing and $20 \mathrm{~min}$ of the seated active or sham tDCS. Participants were repositioned on the force platform with a blindfold and vibration devices (A) Three consecutive trials were performed all consisting of quiet standing (variable duration to prevent predictability of vibration onset, $8-15 \mathrm{~s}$ ), $15 \mathrm{~s}$ of vibration and $25 \mathrm{~s}$ of recovery during which ground reaction forces and torques were recorded. (B) The center of pressure (COP) served as a surrogate for the trajectory of center of mass. A representative example of the COP signal of one participant during the three experimental phases (i.e., quiet standing, vibration and recovery phases) are shown.

Fig. 2. Postural steadiness for the sham (open circles) and active (filled circles) cerebellar tDCS groups during $60 \mathrm{~s}$ of quiet standing and across three consecutive experimental trials. With $\mathrm{COP}_{\mathrm{DISP}}(\mathrm{A}), \mathrm{COP}_{\mathrm{SD}}(\mathrm{B})$ and $\mathrm{COP}_{\mathrm{PATH}}(\mathrm{C})$ for the baseline, vibration and recovery phases, respectively. Negative displacement indicates a backward shift of COP, while positive displacement indicates a forward shift of COP.

Fig. 3. Illustrates current modelling results: (A) lateral views: left image = right hemisphere (B) dorsal (left) and ventral (right) views (C) anterior (left) and posterior (right) views. Electric field intensity (EFI: Volts/meter) is shown. 


\section{Tables}

Table 1 Overview of the mean reported adverse effects of each condition following completion of the session.

\begin{tabular}{llll}
\hline Adverse effects & SHAM & ACTIVE & P-value \\
\hline Headache & $1.00 \pm 0.00$ & $1.14 \pm 0.52$ & 0.31 \\
Neck Pain & $1.07 \pm 0.25$ & $1.07 \pm 0.26$ & 0.96 \\
Scalp Pain & $1.07 \pm 0.25$ & $1.07 \pm 0.26$ & 0.96 \\
Tingling & $1.20 \pm 0.40$ & $1.79 \pm 0.77$ & $0.02^{*}$ \\
Itching & $1.20 \pm 0.40$ & $1.29 \pm 0.59$ & 0.66 \\
Burning Sensation & $1.13 \pm 0.34$ & $1.29 \pm 0.59$ & 0.41 \\
Skin Redness & $1.07 \pm 0.25$ & $1.00 \pm 0.00$ & 0.34 \\
Sleepiness & $1.33 \pm 0.70$ & $1.29 \pm 0.59$ & 0.85 \\
Trouble concentrating & $1.00 \pm 0.00$ & $1.00 \pm 0.00$ & 1.00 \\
Acute mood change & $1.00 \pm 0.00$ & $1.07 \pm 0.26$ & 0.69 \\
\hline
\end{tabular}




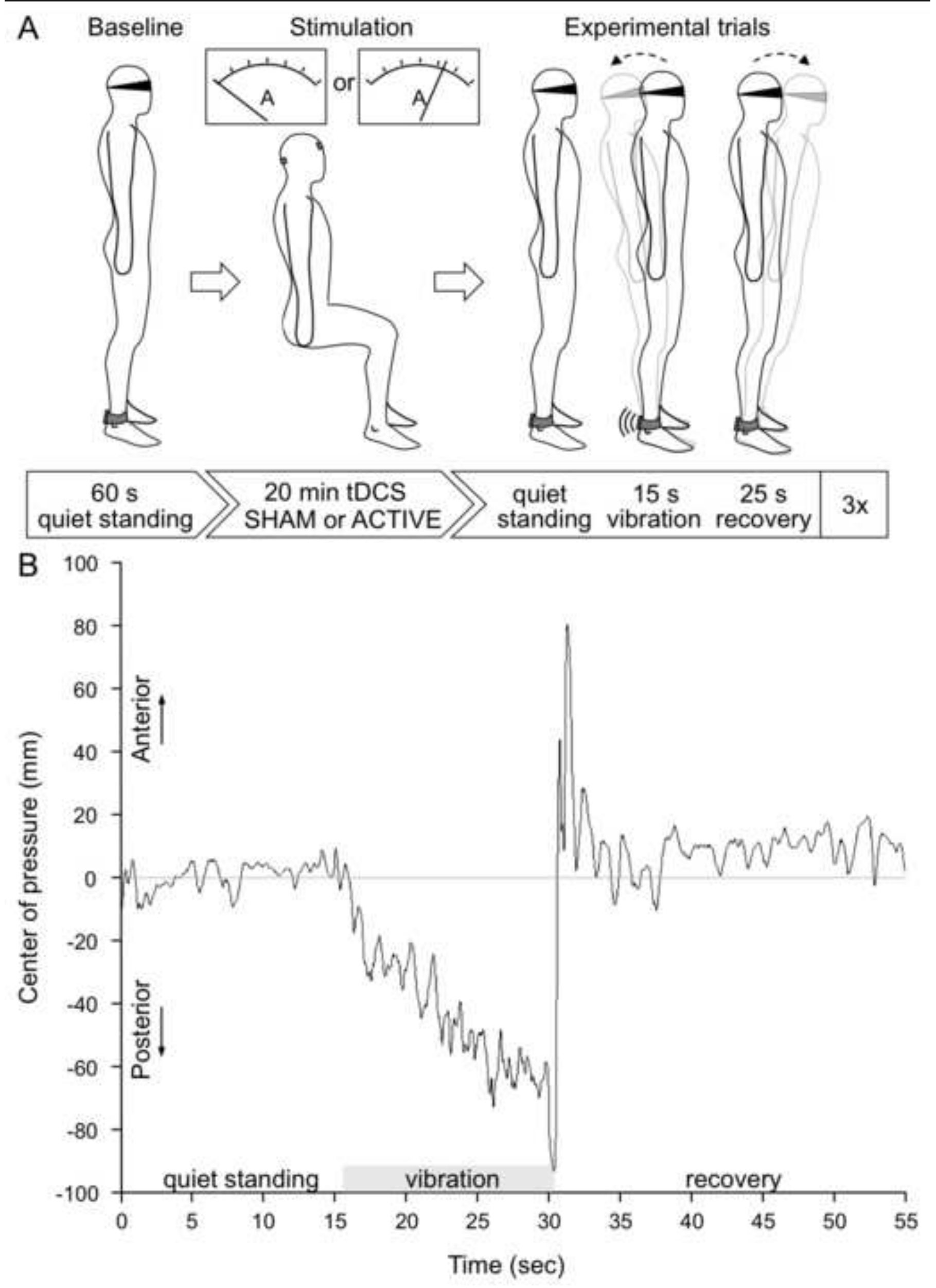




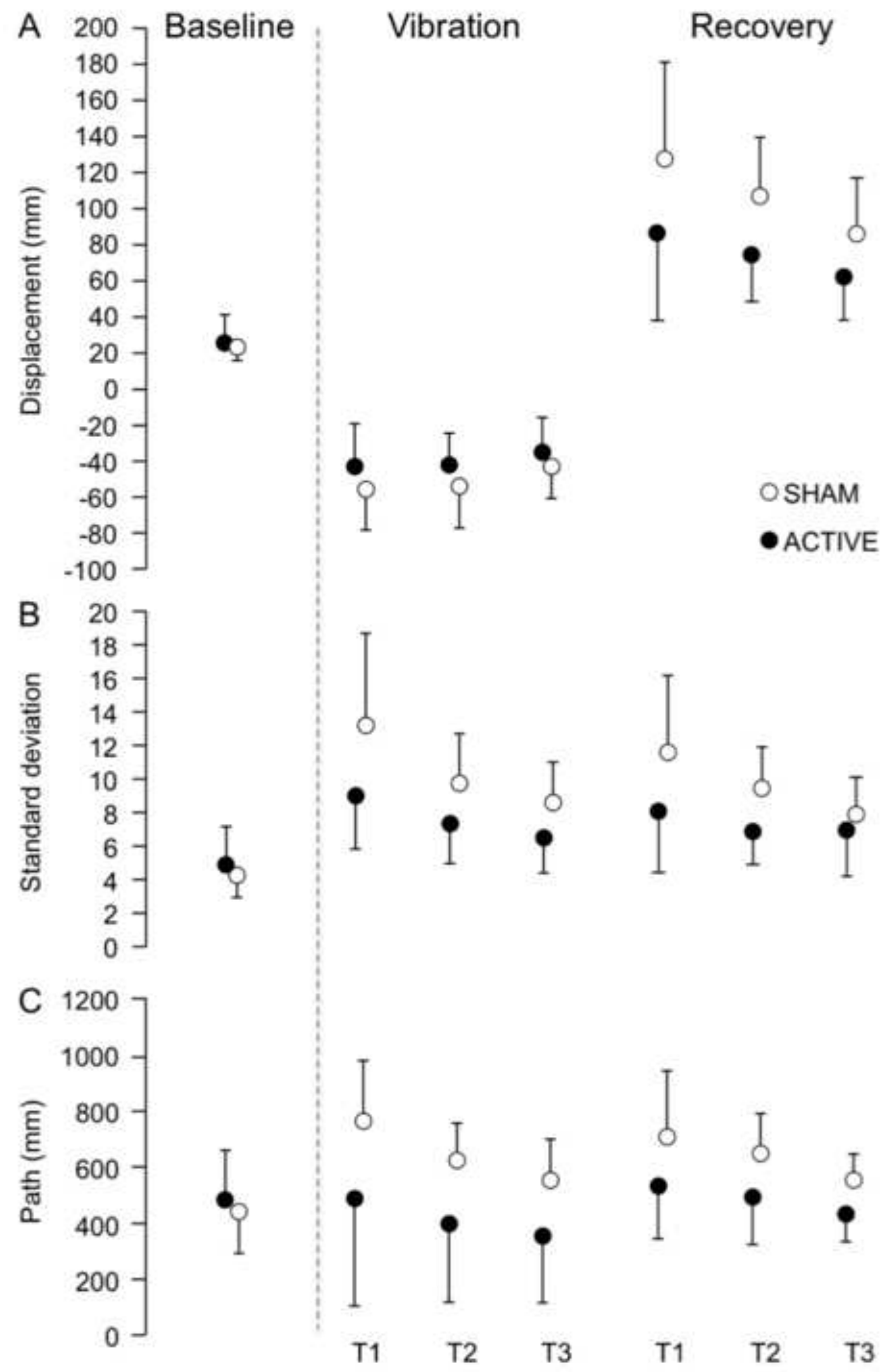



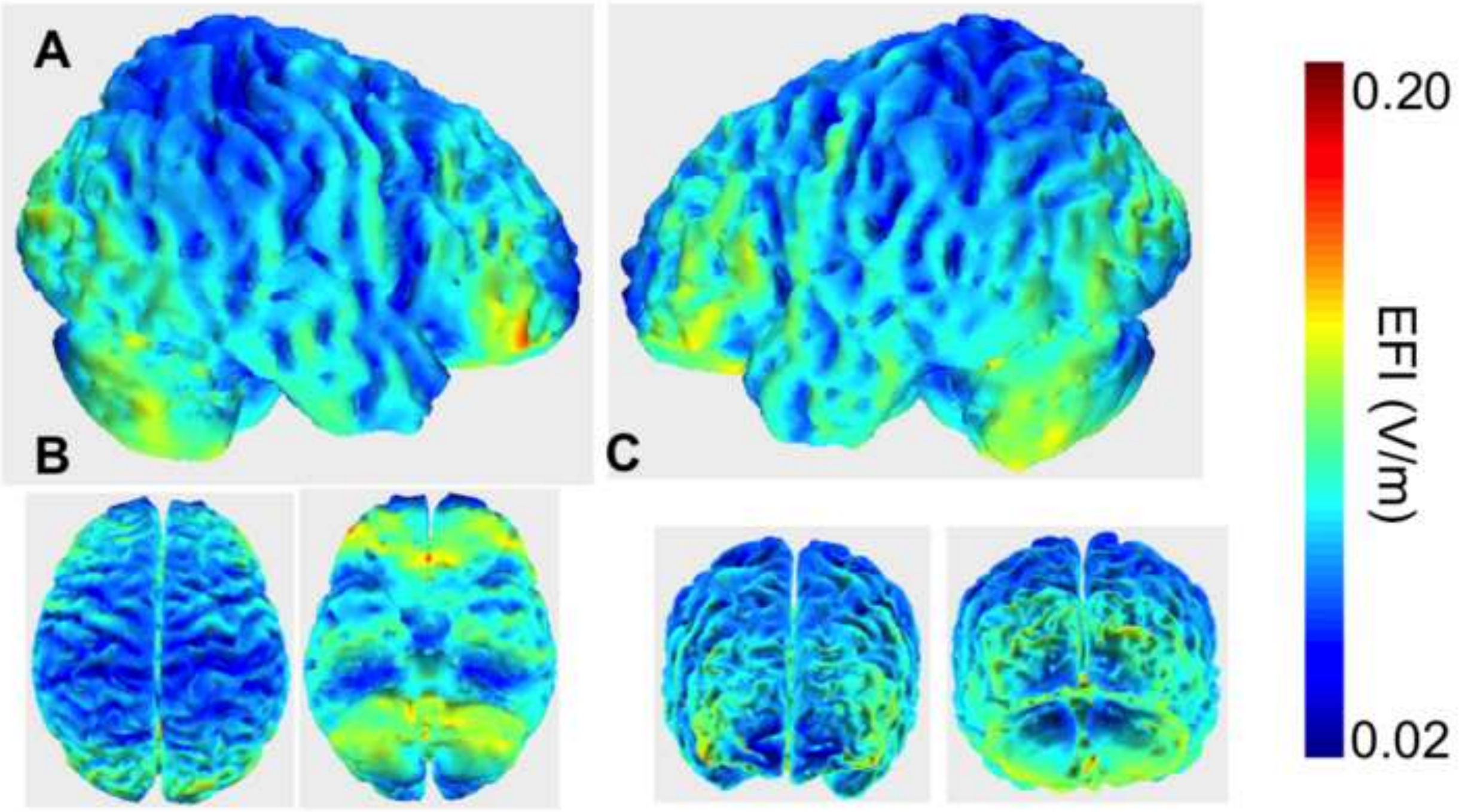